solution was employed throughout the operation, some two to three pints being utilised.

On the following day the patient's condition was much improved; the drainage-tube was replaced by a gauze plug on the third day and the latter was removed on the fourth day. On the second day after the operation pain in the right side of the chest was complained of and examination led to a diagnosis of "dry pleurisy" at the right base and "pneumonia" involving the left lower lobe; the chest was strapped. The temperature on the third day was $98^{\circ}$ and the pulse-rate was 84 per minute. From this date the temperature became irregular, varying from $97^{\circ}$ to $102 \cdot 8^{\circ}$. The bowels were well opened on the third day and the patient enjoyed a fish diet on the eighth day. An increasing area of dulness was detected on the right side of the chest and on the eighteenth day this reached the middle of the scapula posteriorly; a diagnosis of probable "empyema" was made. The signs at the left base gradually cleared up. On the twentieth day an aspirating needle inserted through the ninth right intercostal space in the posterior axillary line withdrew a pint of very offensive pus mixed with gas ; accordingly on the following day a portion of the ninth rib was resected. The general pleural cavity was shut off by adhesions, the dome of the diaphragm being considerably raised, and an incision through the latter revealed a large abscess cavity between the liver and diaphragm, from which another pint of pus was evacuated ; a large drainage-tube was inserted and the wound partly closed. The pus yielded a pure culture of bacillus coli. As the patient's temperature still continued to fluctuate, subcutaneous injections of anti-coli serum were given on the twenty-fifth and two following days; the temperature assumed a lower level. On the twenty-ninth day the patient received a subcutaneous injection of "bacillus coli vaccine" prepared in the St. Thomas's Hospital clinical laboratory by Dr. L. S. Dudgeon. The temperature reached the normal on the thirty-first day but commenced to rise again on the thirty-fourth day, with the advent of an urticarial (serum) rash. The abscess cavity drained well, the tube being gradually shortened and replaced by one of smaller calibre on the forty-second day; another injection of vaccine was also administered. The temperature continued to fluctuate, but gradually it assumed a lower level, a free discharge of offensive pus continuing. The patient got up and sat in a chair for a few hours on the fifty-third day and received another injection of vaccine on the fifty-sixth day. A troublesome cough developed and the temperature rising the patient had to take to his bed again. Examination of the chest revealed an area of dulness at the right base, reaching as high as the middle of the scapula; breath sounds were absent over this area, vocal fremitus was much diminished, and vocal resonance was not obtainable. Aspiration through the ninth space in the scapular line revealed the presence of pus. On the following day a portion of the tenth rib was resected and another large subphrenic collection of pus was evacuated and the cavity was drained; no communication between this abscess cavity and the former could be demonstrated. A fourth injection of vaccine was given on the sixty-fourth day ; the temperature reached the normal level on the sixtyninth day and remained settled. From this date the patient's general condition rapidly improved. Some 14 days later he was allowed to get up morning and evening. The drainage-tubes were gradually shortened and the amount of discharge rapidly diminished and lost its offensive character. The bowels were kept well open throughout the treatment of the case.

On Jan. 28th, 1908, some three and a half months after the accident, the patient was sent to a convalescent home. A final note on the case reads as follows: "Abdominal wounds quite sound -anterior sinus of chest almost closed, posterior sinus is formed by a narrow track some five inches in length - there is no trouble with the bowels." The patient was again examined on his return from the convalescent home four weeks later; he had put on weight-both sinuses had quite closed and he experienced no discomfort whatever. Some five months after the accident he was allowed to start light work.

This case presents several points of interest. First, the comparative rarity of the accident. Secondly, the difficulties presented in the diagnosis of a "sub-phrenic collection of pus," the physical signs suggesting the presence of an empyema, rather than of pus below the diaphragm.
Thirdly, the beneficial effect of the anti-coli serum and bacillus coli vaccine which showed itself in an attempt to stop the spread of the septic process to a great extent, and in the rapid recovery of the patient and rapid healing of the sinuses after evacuation of the pus.

In conclusion, I must express my thanks to Mr. Ballance for his kindness in allowing me to conduct the treatment of the case and for permission to publish these notes. St. Thomas's Hospital.

\section{A METHOD OF PERFORMING ABDOMINO- PERINEAL EXCISION FOR CARCINOMA OF THE RECTUM AND OF THE TERMINAL PORTION OF THE PELVIC COLON.}

By W. ERNEST MILES, F.R.C.S. ENG., L.R.C.P. LOND, SURGFON TO THE CANCER HOSPITAL, BROMPTON, S.W., AND 'TO THE GORDON HOSPITAI, FOR DISEASES OF THE RECTUM, VAUXHALL BRIDGE-ROAD, S.W.

REMONaL of the rectum by a combined abdominal and perineal operation was first performed by Czerny in 1884 . Since that time several other surgeons, notably Maunsell, Chaput, Gaudier, Challot, Weir, Boechal, Giordino, Quénu, Reverdin, Tuttle, Gant, Mathews, Sir Charles Ball, Wallis, and Aldrich-Blake have employed the method with certain modifications of procedure and with varying success in regard to mortality. So far as I have been able to gather from the literature of the subject, however, the technique of these operators seems to have failed in one important respectnamely, the complete eradication of the zone of upward spread of cancer from the rectum, whereby the chance of recurrence of the disease, above the field of operation, can be diminished if not entirely obviated.

Until the close of the year 1906 I had relied solely upon the perineal methods of excision of the rectum, but $I$ found that, even after the most complete and extensive removal possible, recurrence of the disease was a rule to which there were few exceptions. In fact, from a personal experience of 57 such operations, my records showed the disquiet. ing fact that recurrence took place within periods ranging from six months to three years in 54 instances. Moreover, I found that although perfection of technique had reduced the proportion of recurrences in the immediate vicinity of the field of operation, recurrence nevertheless appeared in situations that were beyond the scope of a removal from the perineum. Post-mortem examination showed that these situations were (a) the pelvic peritoneum, $(b)$ the pelvic mesocolon, and $(c)$ the lymph nodes situated over the bifurcation of the left common iliac artery. Bearing these facts in mind I made observations upon the course taken by the upward spread of cancer among patients who had died from inoperable cancer of the rectum and found that the disease invariably extended by continuity of tissue along the parietal attachment of the pelvic mesocolon and in the adjacent parietal peritoneum for about one inch on either side of it as far as the group of lymph nodes situated over the bifurcation of the common iliac artery. In one case the disease extended by continuous spread to the suprarenal capsule of the left kidney. In all the cases thus examined the infiltration of the parietal border of the pelvic mesocolon had caused shrinkage of the pelvic mesocolon itself, whereby the pelvic colon appeared to be bound down, a condition which readily explains the difficulty in obtaining a satisfactory spur when performing colostomy in an advanced case of cancer of the rectum.

From these observations it is obvious that the abovementioned structures constitute the zone of the upward spread of cancer from the rectum, the removal of which is just as imperative as is the thorough clearance of the axilla in cases of cancer of the breast if freedom from recurrence is to be hoped for. The appreciation of this important fact induced me two years ago to abandon the perineal methods of excision of the rectum as inadequate for the purpose of obviating recurrence and to endeavour, by utilising an abdominal method of procedure, to bring the operation of excision of the rectum into line with those methods of performing abdominal 
iysterectomy known as the Wertheim and the Krönig-

Wertheim.

The study of the spread of cancer from the rectum has led me to formulate certain essentials in the technique of the ojeration which must be strictly adhered to if satisfactory results are to be obtained-namely: (1) that an abdominal anus is a necessity ; (2) that the whole of the pelvic colon, with the exception of the part from which the colostomy is made, must be removed because its blood-supply is contained in the zone of upward spread; (3) that the whole of the pelvic mesocolon below the point where it crosses the common iliac artery, together with a strip of peritoneum at least an inch wide on either side of it, must be cleared away; (4) that the group of lymph nodes situated over the bifurcation of the common iliac artery are in all instances to be removed ; and lastly (5), that the perineal portion of the operation should be carried out as widely as possible so that the lateral and downward zones of spread may be effectively extirpated.

\section{The Operation.}

This is one of the most formidable operations in surgery, entailing, as it does, the removal of practically the whole of the pelvic colon as well as the rectum. I have now performed it 12 times with a mortality of $41 \cdot 6$ per cent. Nine of the patients were males, of whom four died, and three were females, of whom one died. The surviving seven patients are all at present free from recurrence, the first three having been operated upon in January, March, and May of 1907 respectively.

Preparation of the patient. - In all cases in which there is marked stenosis of the bowel, a preliminary left iliac colostomy should be performed at least two weeks before the operation. If there be no abdominal distension and if the lumen of the bowel be not much encroached upon the colon should be thoroughly emptied of its contents by the aid of enemata and mild purgation.

Method of operating.--The patient having been placed in an exaggerated Trendelenburg posture, a median incision is made from the umbilicus to the symphysis pubis and a self-retaining abdominal claw retractor is placed in position. The pelvic colon is then drawn out into the wound and a left iliac colostomy, if one does not already exist, is established in the usual situation through a second incision. I usually do this by simply pushing a loop of the nppermost part of the pelvic colon through an incision in the abdominal wall and fixing it in position in the ordinary way. Some operators advocate first dividing the bowel and then establishing the colostomy from the proximal end only, but I think that by so doing there is a greater tendency to protrusion afterwards. As soon as the knuckle of colon has been fixed in position in the manner indicated, the pelvic colon is divided transversely about two inches below it and both ends are closed firmly by means of purse-string sutures. The closure of the distal end should be done carefully, so as to avoid leakage from that part of the bowel during the subsequent steps of the operation. The peritoneal covering of the pelvic mesocolon is then divided transversely backwards on either side to the point where the pelvic mesocolon crosses the common iliac artery, the incisions extending into the parietal peritoneum for the distance of about one inch. The inferior mesenteric artery is then ligatured below the point where it gives off its uppermost branch to the pelvic colon. When this has been done the pelvic mesocolon itself is divided completely as far backwards as its parietal attachment, the inferior mesenteric artery being divided below the point of ligature. The upper and remaining portion of the pelvic mesocolon is now turned upwards for the distance of an inch or more and all the cellular tissue containing the group of lymph nodes situated over the bifurcation of the left common iliac artery dissected carefully away. All bleeding vessels in the cut edge of the pelvic mesocolon are caught and tied. The remainder of the operation is now practically bloodless and should be rapidly proceeded with. The peritoneum is next divided by incisions which extend downwards into the pelvis, one on either side of the parietal attachment of the pelvic mesocolon and at least one inch distant from it, until the recto-vesical pouch is reached, when they are carried round the lateral aspects of the pelvic wall to meet again in front just behind the base of the bladder. In the female the left broad ligament should be detached, together with the left ovary and tube. This having been done, the pelvic mesocolon together with the adjacent strip of peritoneum on either side of it is detached from the hollow of the sacrum. By keeping close to the anterior sacral ligaments, the cellular tissue containing the $\cdot$ lymph nodes in that situation is detached with the pelvic mesocolon in one piece. This separation is continued downwards in the middle line as far as the sacro-coccygeal articulation. A similar method of blunt dissection is then carried out anteriorly, by which means the bladder is detached as far as the prostate gland. Attention is now paid to the separation of the lateral aspects of the rectum and it is here that great care must be exercised to avoid injuring the left ureter which adheres closely to the peritoneum as it skirts the wall of the pelvis. When the ureter has been defined it should be carefully freed as far as the base of the bladder. On the right side the ureter need not be seen. The dissection is then carried downwards on either side and the lateral ligaments of the rectum are divided with scissors. In these structures the middle hæmorrhoidal arteries are found but seldom require a ligature. This lateral dissection is carried down to the upper surface of the levatores ani. When the rectum has thus been freed on all sides as far as the points indicated, the whole of the detached structures are crowded down into the pelvis and covered with sterilised gauze. Provided that the divided end of the colon has been firmly closed considerable pressure may be made upon it from above without fear of leakage. The edges of the pelvic peritoneum are now sutured so as to re-establish the pelvic floor. This having been done, the pelvis is thoroughly flushed with saline solution and the abdominal wound closed. The patient is now turned over and placed in the right lateral and semi-prone position so that the perineal portion of the operation can be proceeded with. A purse-string suture having been inserted round the anus to prevent escape of its contents, a transverse incision is made at the level of the sacro-coccygeal joint and from the centre of this a median incision is carried down to within an inch of the anus. From the lowermost extremity of the latter a semicircular incision is made round the anus on either side, meeting anteriorly at the central point of the perineum. These last incisions should include as wide an area of skin as possible so as to insure excision of the zone of downward spread. After reflecting the skin on either side to the requisite extent, the coccyx is removed and the interval between the levatores ani defined. These muscles should be divided as far outwards as their origin from the "white line" so as to include the lateral zone of spread. The remainder of the fascia propria of the rectum is then detached from the lower part of the sacrum when the pelvic colon and the rectum, which lie loose in the hollow of the sacrum, can be drawn out of the wound. The lower part of the rectum is then dissected from the prostate or the vagina, as the case may be, care being taken to remove the cellular tissue in its vicinity as freely as possible. The resulting chasm is then freely irrigated with saline solution and all bleeding vessels are tied. Finally, the skin margins are brought together with sutures and a large drainage-tube is inserted in the anterior and the posterior extremities of the median incision. The patient is now turned upon his back and as the final step of the operation a small opening is made into the extruded bowel to allow of the escape of flatus.

The operation thus performed takes from an honr and a quarter to an hour and a half and, so far as my experience goes, the patient suffers from no more shock than after an ordinary perineal excision.

The mortality after the operation appears to be high, but it must be remembered that the disease for which it is performed claims a mortality of 100 per cent. if left alone and of over 90 per cent. from recurrence when operated upon by the perineal methods. The causes of death in my cases have been : one from hypostatic congestion of the lungs, one from strangulation of a knuckle of small intestine which had become herniated through a rent in the peritoneal pelvic floor, one from gangrene of the stump of the pelvic colon below the colostomy, and two from peritonitis. With the exception of the first named I regard all these causes of death as preventable with further experience of the operation and with improved technique, and therefore hope that in a future series of cases I shall be able to show a lower degree of mortality than $41 \cdot 6$ per cent.

Upper Wimpole-street, $W$. 